\title{
The association of paramedic rapid sequence intubation and survival in out-of-hospital stroke
}

\author{
Pieter Francsois Fouche, ${ }^{\oplus 1}$ Karen Smith, ${ }^{2}$ Paul Andrew Jennings, ${ }^{3}$ Malcolm Boyle, ${ }^{4}$ \\ Stephen Bernard ${ }^{5}$
}

Additional material is published online only. To view please visit the journal online (http://dx.doi.org/10.1136/ emermed-2019-208613).

${ }^{1}$ Department of Community Emergency Health and Paramedic Practice, Monash University, Frankston, Victoria, Australia

${ }^{2}$ Research and Evaluation, Ambulance Victoria, Melbourne, Victoria, Australia

${ }^{3}$ Ambulance Victoria, Doncaster,

Victoria, Australia

${ }^{4}$ Paramedicine, Griffith University School of Medicine, Southport, Queensland, Australia

${ }^{5}$ Research and Evaluation, Ambulance Victoria, Melbourne, Victoria, Australia

Correspondence to Mr. Pieter Francsois Fouche: Pieter.Fouche@monash.edu

Received 26 March 2019 Revised 4 May 2019 Accepted 10 May 2019

\begin{abstract}
Introduction Ambulance transport of patients with stroke is common, with rapid sequence intubation (RSI) to secure the airway used regularly. Randomised controlled trial evidence exists to support the use of RSI in traumatic brain injuries (TBIs), but it is not clear whether the RSI evidence from TBI can be applied to the patient with stroke. To this end, we analysed a retrospective stroke dataset to compare survival of patients with RSI compared with patients that did not receive $\mathrm{RSI}$.
\end{abstract}

Methods This study was a retrospective analysis of 10 years of in-hospital and out-of-hospital data for all patients with stroke attended by Ambulance Victoria, in Victoria Australia. Generalised boosted logistic regression was used to predict propensity scores, with initial vital signs, age and demographic variables as well as measures of illness severity and comorbidity included in the prediction model. This analysis employed a 1:1 nearest-neighbour matching which was applied to generate a dataset from which we calculated the OR of survival to hospital discharge of patients receiving RSI versus no-RSI. The sensitivity of these results to unmeasured confounding was assessed with deterministic sensitivity analysis.

Results The propensity score-matched cohort showed a decreased survival for RSI in strokes with an OR 0.61 (95\% Cl 0.45 to $0.82 ; p=0.001$ ) when compared with no-RSI. A subgroup analysis showed no significant survival difference for ischaemic strokes: OR 0.66 (95\% $\mathrm{Cl} 40$ to 1.07; $p=0.09$ ). The survival for haemorrhagic stroke was OR 0.60 (95\% Cl 0.41 to $0.90 ; p=0.01$ ) lesser for RSI. Results were likely robust to unmeasured confounding and missing data.

Conclusions Our retrospective analysis shows a decrease in survival when RSI is utilised by paramedics for stroke. Since RSI is commonly used for strokes, controlled trial evidence to support this practice is urgently needed.

\section{INTRODUCTION}

Strokes accounts for $10 \%$ of deaths worldwide, and almost $5 \%$ of all disability adjusted life years. Rapid sequence intubation (RSI) is a commonly used procedure to secure the airway in patients with stroke that uses sedative and paralytic drugs to facilitate endotracheal intubation. It is not clear what proportion of out-of-hospital strokes receive RSI, but Meyer et al reported that 55\% of their out-of-hospital haemorrhagic strokes received RSI. ${ }^{2}$ The Meyers study suggests that RSI use might be high in haemorrhagic strokes, but it is not known

\section{Key messages}

What is already known on this subject

- Rapid sequence intubation (RSI) is used by paramedics to secure the airway in strokes. The only evidence to support such use is an out-of-hospital randomised controlled trial of traumatic brain injuries. Brain trauma differ from stroke, and it is not clear that the evidence from this head injury trial can be extrapolated to stroke.

\section{What this study adds}

- This propensity score-matched cohort study of out-of hospital RSI by paramedics is the first to show decreased survival for RSI in strokes. Furthermore, this study demonstrates a considerably larger decrease in systolic blood pressure for those that receive RSI, compared with no-RSI. Additional derangements in carbon dioxide, oxygen, pulse rate and respiratory rate found here could explain this decreased survival in stroke.

how common strokes are in those that receive RSI. A recent Australian out-of-hospital study showed that strokes form a substantial proportion (36.6\%) of RSI undertaken by paramedics. ${ }^{3}$

While RSI is commonly used by paramedics in stroke, no high-quality evidence for RSI in stroke currently exist. ${ }^{3}$ An out-of-hospital randomised trial that compared paramedic RSI for traumatic head injury showed favourable neurological outcome compared with in-hospital RSI. ${ }^{4}$ However, it is not clear that the evidence from this trial can be generalised to strokes, as there are significant differences between patients with the stroke and traumatic brain injury (TBI). One such difference is the impact of mechanical ventilation. Mechanical ventilation accompanies intubation and could decrease cardiac output. For example, intracranial haemorrhages frequently have decreased cardiac output, which is less typical in brain trauma. ${ }^{5}$ This suggests that mechanical ventilation after Intubation could influence survival differently when TBI is compared with strokes because of this difference in cardiac output. On average, TBIstroke. Since age is associated with increased mortality from nosocomial infections after mechanical ventilation, ${ }^{6}$ and keeping in mind that strokes tend to be older than 
patients with TBI, intubation in RSI could cause more infections in stroke compared with brain trauma.

If the evidence from brain trauma cannot be used as a basis for stroke airway management, then research to justify stroke RSI is needed. The objective of this study to analyse a retrospective stroke dataset to compare survival of patients with stroke who received RSI compared with patients that did not receive RSI by paramedics.

\section{METHODS}

\section{Study setting and data sources}

Victoria has nearly 6.5 million residents serviced by a single two-tier emergency medical service, Ambulance Victoria. The two tiers consist of Mobile Intensive Care Paramedics (MICA) and advanced life support paramedics. Only MICA paramedics are authorised to provide RSI to patients that have a Glasgow Coma Scale (GCS) of less than 10 due to head trauma, non-TBIs, respiratory failure, severe hyperthermia, severe uncontrolled pain and airway burns. ${ }^{7}$ Suxamethonium is the primary paralytic, with pancuronium used to maintain paralysis. Midazolam, morphine/midazolam infusions, atropine, ketamine and fentanyl are available to assist RSI. ${ }^{7}$ RSI is authorised when transport time is more than $10 \mathrm{~min}$ to the nearest suitable emergency department. This study analysed data from 131 hospitals and clinics in Victoria, Australia from the 10-year period: 1 January 2008 to 31 December 2017. The Centre for Victorian Data Linkage provided all in-hospital patient records via the Victorian Admitted Episodes and Victorian Emergency Minimum dataset and Ambulance Victoria provided all out-of-hospital data. In-hospital and out-of hospital datasets were merged using deterministic linkage. Monash University Human Research Ethics Committee gave ethics approval for this study (Ref. No. 8618).

\section{Selection of study cohort}

This study included all patients of any age that were treated and transported by Ambulance Victoria, with a final hospital diagnosis of stroke. We excluded all cases with traumatic intracranial injury, transient ischaemic attack and strokes that could not be classified as either haemorrhagic or ischaemic. Instances of stroke were identified by the Australian modification of ICD10 codes recorded for a particular episode of care, and incorporated codes: I60, I61, I62.9 and I63. These Tenth revision of the International Statistical Classification of Diseases and Related Health Problems (ICD10) codes served as an operational definition of the strokes included in our cohort. We included all strokes, whether they were primary or secondary, for a particular episode of care. The Centre for Victorian Data Linkage selected all patients with stroke codes, regardless of ambulance transport or not. These patients with stroke were then linked to the Ambulance Victoria out-of-hospital dataset to select a cohort that had a stroke and were transported by ambulance.

\section{Predictors and outcomes}

The primary outcome was survival to hospital discharge, which is defined as the patient being discharged from hospital alive and is identified from the discharge disposition at hospital separation. Variables from in-hospital records and the Ambulance Victoria datasets served as potential predictors and include demographic, treatment, baseline observations, scene and transport time intervals, pathologies, length of stay and outcomes.

Prognostic risk influences the choice to use intubation, causing confounding by indication. ${ }^{8}$ Previous work shows that illness severity and comorbidity are important confounders-by-indication in non-TBIs. ${ }^{3}$ An effective way to account for confounding by indication is to use illness severity and comorbidity risk adjustment. ${ }^{9}$ To account for comorbidity, we calculated and adjusted for the Walraven-Elixhauser score. ${ }^{10}$ Illness severity was quantified using the GCS. While the GCS was not designed as a stroke severity score, it is similarly predictive of in-hospital mortality as National Institute of Health Stroke Scale (NIHSS) in patients with stroke. ${ }^{11}$ To achieve proper risk adjustment, we added the Walraven-Elixhauser comorbidity score and the initial paramedic measured GCS in the propensity score model. We specified a priori that age, initial pulse rate, blood pressure, respiratory rate, GCS, $\mathrm{SPO}_{2}$, blood glucose level, comorbidity, the year of RSI, sex and type of stroke were important predictors and these were included in our propensity prediction model if there were sufficient data (more than 90\%). We did not adjust for any in-hospital treatments, as we believe that RSI can cause the likelihood of in-hospital interventions, and such adjustments would be on a mediator variable, which would introduce bias. ${ }^{12}$ Selection of propensity score prediction variables was based on stroke literature and a causal directed acyclic graph (online supplementary figure S1).

\section{Definitions}

Strokes are defined by ICD10-AM codes and we further subtyped strokes for ischaemic and haemorrhagic strokes. Ischaemic strokes include cerebral infarction and haemorrhagic strokes comprise subarachnoid heammorhage, intracerebral heammorhage and other intracranial non-traumatic heammorhage. RSI is defined as the attempted or successful placement of an endotracheal tube in the trachea after receiving a paralytic agent, with or without additional medications. Successful placement of endotracheal tube in the trachea was confirmed using clinical means and end-tidal $\mathrm{CO}_{2}$ waveform. Hypotension was defined as a systolic blood pressure less than $90 \mathrm{~mm} \mathrm{Hg}$, and normocapnia as an end-tidal $\mathrm{CO}_{2}$ of between 35 and $45 \mathrm{~mm} \mathrm{Hg}$. Hypoxia was defined as a $\mathrm{SPO}_{2}$ reading of less than $90 \%$.

\section{Statistical analysis}

Stata V.14 (Stata Corp, College Station, Texas, USA) was used in this analysis. Categorical variables are presented as frequencies, and continuous variables as means with SD. Categorical variables were compared with the $\chi^{2}$ test and continuous predictors with the t-test or Wilcoxon rank-sum test. Hypothesis tests were two-sided, with a significance level of $\mathrm{p}<0.05$.

\section{Propensity score matching}

This analysis used propensity score matching to adjust for confounding bias. A generalised boosted logistic model was used to predict propensity scores. This boosted model implements the boosting algorithm described by Hastie, Tibshirani and Friedman, and is a re-interpretation of adaptive boosting, which was modified into the likelihood framework. ${ }^{13}$ We performed a 1:1 nearest-neighbour match for each patient that had received RSI within a maximum calliper of 0.1 of the propensity score. The propensity matched sample was used to conduct a multilevel logistic regression to account for the clustering effect of matching and hospitals. All selected covariates were added to the fixed effect portion, and hospital was added as a random effect. All variables that were in the propensity score model were used in the logistic regression, and results are reported as ORs. Model fit of the analysis of the matched dataset was assessed with Hosmer and Lemeshow goodness-of-fit test, and the boosted model with pseudo- $\mathrm{R}^{2}$. To assess the performance of 


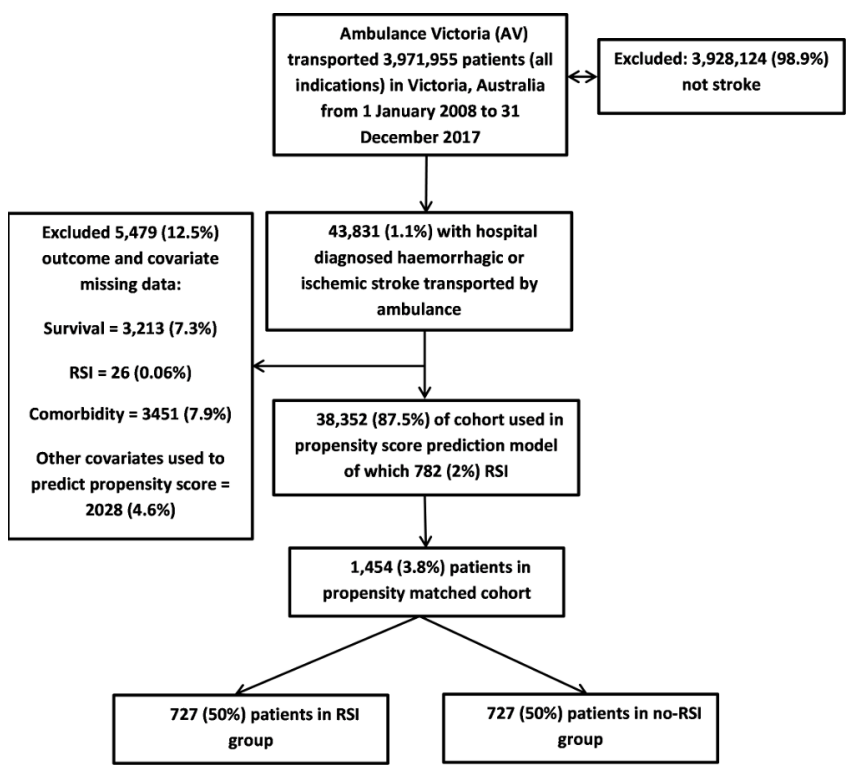

Figure 1 Patient selection for a cohort of RSI in stroke. RSI, rapid sequence intubation.

the propensity score matching, baseline and post-match variables were compared using standardised differences, where a difference less than or equal to 0.1 was considered insignificant. Furthermore, we compared RSI versus no-RSI for changes in systolic blood pressure, respiratory rate, pulse oximetry, GCS and pulse. These vital-sign changes were calculated by subtracting the final on-scene value from the first measurement on arrival.

\section{Sensitivity analysis}

We deleted missing covariate observations for the propensity score prediction model and main analysis and compared these results to an analysis with imputed missing covariates. We did not impute survival or RSI itself. Additionally, we tested the robustness of the results to unmeasured confounding using a deterministic sensitivity analysis. ${ }^{14}$ Previous research showed that a large source of unmeasured confounding is likely to be unaccounted for by illness severity. ${ }^{15}$ Consequently, we calculated the impact on survival of $10 \%$ to $100 \%$ additional prevalence of unmeasured illness severity in the RSI group, compared with an extra 5\% in the no-RSI group. Furthermore, we anticipated missing covariates, and we imputed missing data using multivariate normal regression for each covariate and treatment group separately. No imputations of survival were completed and were deleted from all analyses. We deleted missing covariate observations for the main analysis and compared these results to an analysis with imputed missing observations.

\section{RESULTS}

This cohort of stroke transported by ambulance included 43831 patients in Victoria, Australia (figure 1) of which 882 $(2.0 \%)$ received RSI. Baseline and in-hospital characteristics are compared in table 1 . Of the 38352 with complete data, 12708 (33.1\%) were diagnosed with haemorrhagic stroke and 26996 $(70.4 \%)$ with ischaemic stroke, with 1352 (3.5\%) diagnosed with both stroke types. Of those who received RSI, suxamethonium was utilised in 777 (99.4\%) of patients and pancuronium in $631(80.7 \%)$ of patients. Fentanyl was used in $750(95.9 \%)$ of the cohort; ketamine in 20 (2.6\%); midazolam in 752 (96.2\%) and midazolam/morphine infusions in 627 (80.2\%). The overall
Table 1 Patient, demographic, prognostic and in-hospital factors in the full cohort of stroke

\begin{tabular}{|c|c|c|c|}
\hline \multirow[b]{2}{*}{ Characteristic } & \multicolumn{3}{|c|}{ Patients, No. (\%) } \\
\hline & $\begin{array}{l}\text { Total } \\
(\mathrm{n}=38352)\end{array}$ & RSI $(n=782)$ & $\begin{array}{l}\text { No-RSI } \\
(n=37570)\end{array}$ \\
\hline \multicolumn{4}{|l|}{ Demographic } \\
\hline Age, mean (SD), years & $73.0(15.2)$ & $65.2(15.6)$ & $73.1(15.2)$ \\
\hline \multicolumn{4}{|l|}{ Sex } \\
\hline Male & $19662(51.3)$ & $382(48.9)$ & $19280(51.3)$ \\
\hline Female & $18690(48.7)$ & $400(51.2)$ & $18290(48.7)$ \\
\hline \multicolumn{4}{|l|}{ IIIness } \\
\hline Haemorrhagic stroke & $12708(33.1)$ & $581(74.3)$ & $12127(32.3)$ \\
\hline Ischaemic stroke & $26996(70.4)$ & $244(31.2)$ & $26752(71.2)$ \\
\hline \multicolumn{4}{|c|}{ IIIness severity/comorbidity, mean (SD) } \\
\hline $\begin{array}{l}\text { Elixhauser comorbidity } \\
\text { score* }^{*}\end{array}$ & $20.8(7.4)$ & $18.1(7.4)$ & $20.8(7.4)$ \\
\hline Initial Glasgow Coma Scale & $13.2(3.0)$ & $6.1(3.3)$ & $13.4(2.8)$ \\
\hline \multicolumn{4}{|l|}{ Observations, mean (SD) } \\
\hline Initial pulse rate & $83.2(19.8)$ & $88.3(27.7)$ & $83.1(19.6)$ \\
\hline Final pulse rate & $81.9(19.1)$ & $99.0(22.6)$ & $81.6(18.7)$ \\
\hline Initial systolic blood pressure & $151.1(32.8)$ & $162.6(44.3)$ & $150.8(32.5)$ \\
\hline Final systolic blood pressure & $149.4(30.1)$ & $145.3(32.2)$ & $149.5(30.0)$ \\
\hline Initial respiratory rate & $17.7(4.8)$ & $17.8(7.9)$ & $17.7(4.7)$ \\
\hline Final respiratory rate & $17.1(4.3)$ & $12.0(7.2)$ & $17.2(4.1)$ \\
\hline Initial $\mathrm{SPO}_{2}$ & $95.6(5.6)$ & $93.6(10.5)$ & $95.7(5.3)$ \\
\hline Final $\mathrm{SPO}_{2}$ & $96.9(3.6)$ & $98.4(4.6)$ & $96.8(3.6)$ \\
\hline \multicolumn{4}{|c|}{ Ambulance time intervals, minutes mean (SD) } \\
\hline Response time & $19.7(21.8)$ & $15.7(16.6)$ & $19.8(21.8)$ \\
\hline Scene time & $22.6(14.2)$ & $57.9(25.0)$ & $21.8(12.9)$ \\
\hline Transport time & $24.0(21.8)$ & $29.8(22.5)$ & $23.9(21.7)$ \\
\hline \multicolumn{4}{|l|}{ Hospital } \\
\hline $\begin{array}{l}\text { Time in intensive care unit, } \\
\text { mean (SD), hours }\end{array}$ & $121.6(164.5)$ & $111.5(137.6)$ & $122.9(167.5)$ \\
\hline $\begin{array}{l}\text { Mechanical ventilation in } \\
\text { intensive care unit, mean } \\
\text { (SD), hours }\end{array}$ & $104.3(153.6)$ & $82.3(112.4)$ & $108.3(159.5)$ \\
\hline $\begin{array}{l}\text { Hospital length of stay, mean } \\
\text { (SD), days }\end{array}$ & $9.1(11.4)$ & $10.1(20.4)$ & $9.1(11.2)$ \\
\hline $\begin{array}{l}\text { Emergency department } \\
\text { length of stay mean (SD), } \\
\text { min }\end{array}$ & $446.7(332.6)$ & $314.5(234.6)$ & $449.9(334.0)$ \\
\hline \multicolumn{4}{|l|}{ Year } \\
\hline 2008 & $3154(8.2)$ & $82(10.5)$ & $3072(8.2)$ \\
\hline 2009 & $3480(9.1)$ & $82(10.5)$ & $3398(9.0)$ \\
\hline 2010 & $3790(9.9)$ & $104(13.3)$ & $3686(9.8)$ \\
\hline 2011 & $3879(10.1)$ & $94(12.0)$ & $3785(10.1)$ \\
\hline 2012 & $3892(10.2)$ & $81(10.4)$ & $3811(10.4)$ \\
\hline 2013 & 3860 (10.1) & $69(8.8)$ & $3791(10.1)$ \\
\hline 2014 & $3112(8.1)$ & $54(6.9)$ & 3058 (8.1) \\
\hline 2015 & $4092(10.7)$ & $56(7.2)$ & $4036(10.7)$ \\
\hline 2016 & 4426 (11.5) & 83 (10.6) & 4343 (11.6) \\
\hline 2017 & 4667 (12.2) & 77 (9.9) & 4590 (12.2) \\
\hline
\end{tabular}

*Scaled to avoid negative values.

RSI, rapid sequence intubation.

intubation success was $97.3 \%$ and first-pass success $89.4 \%$ with no significant change in success over time for overall ( $p=0.50$ for trend) and first pass ( $p=0.07$ for trend). 


\begin{tabular}{|c|c|c|c|}
\hline \multirow[b]{2}{*}{ Characteristic } & \multicolumn{3}{|c|}{ Patients, No. (\%) } \\
\hline & RSI $(n=727)$ & No-RSI $(n=727)$ & $\begin{array}{l}\text { Standardised } \\
\text { Difference }\end{array}$ \\
\hline \multicolumn{4}{|l|}{ Demographic } \\
\hline $\begin{array}{l}\text { Age, mean }(S D) \text {, } \\
\text { years }\end{array}$ & $65.8(15.5)$ & $65.6(16.5)$ & 0.01 \\
\hline Sex & & & 0.04 \\
\hline Male & $356(49.0)$ & $342(47.0)$ & \\
\hline Female & $371(51.0)$ & $385(52.9)$ & \\
\hline \multicolumn{4}{|l|}{ Illness } \\
\hline $\begin{array}{l}\text { Haemorrhagic } \\
\text { stroke }\end{array}$ & $527(72.5)$ & $536(73.7)$ & 0.03 \\
\hline Ischaemic stroke & $241(33.2)$ & $219(30.1)$ & 0.07 \\
\hline \multicolumn{4}{|c|}{ IIIness severity/comorbidity, mean (SD) } \\
\hline $\begin{array}{l}\text { Elixhauser } \\
\text { comorbidity } \text { score }^{*}\end{array}$ & $18.3(7.5)$ & $18.3(7.3)$ & 0.001 \\
\hline $\begin{array}{l}\text { Initial Glasgow } \\
\text { Coma Scale }\end{array}$ & $6.2(3.4)$ & $6.0(3.1)$ & 0.05 \\
\hline \multicolumn{4}{|c|}{ Observations, mean (SD) } \\
\hline Initial pulse rate & $88.5(27.2)$ & $89.1(27.4)$ & 0.02 \\
\hline $\begin{array}{l}\text { Initial systolic } \\
\text { blood pressure }\end{array}$ & $160.2(42.6)$ & $159.3(42.1)$ & 0.02 \\
\hline $\begin{array}{l}\text { Initial respiratory } \\
\text { rate }\end{array}$ & $17.9(7.9)$ & $18.2(7.7)$ & 0.04 \\
\hline Initial $\mathrm{SPO}_{2}$ & $93.6(10.6)$ & $93.1(10.6)$ & 0.04 \\
\hline \multicolumn{4}{|l|}{ Year } \\
\hline 2008 & $73(10.0)$ & $77(10.6)$ & 0.02 \\
\hline 2009 & $76(10.5)$ & $83(11.4)$ & \\
\hline 2010 & $99(13.6)$ & $91(12.5)$ & \\
\hline 2011 & $86(11.8)$ & $77(10.6)$ & \\
\hline 2012 & $76(10.5)$ & $68(9.4)$ & \\
\hline 2013 & $65(8.9)$ & $64(8.8)$ & \\
\hline 2014 & $54(7.4)$ & $56(7.7)$ & \\
\hline 2015 & $51(7.0)$ & $67(9.2)$ & \\
\hline 2016 & 79 (10.9) & $65(8.9)$ & \\
\hline 2017 & $68(9.4)$ & $79(10.9)$ & \\
\hline
\end{tabular}

*Scaled to avoid negative values.

$\mathrm{RSI}$, rapid sequence intubation.

\section{Overall outcomes}

After deleting observations with missing data, the cohort comprised of 38352 transported by ambulance with a stroke of which 30926 (80.6\%) survived to hospital discharge. In an unadjusted regression analysis those that had received RSI had lesser survival of OR 0.08 (95\% CI: 0.07 to $0.1 ; \mathrm{p}<0.001$ ) compared with those patients that did not receive RSI. Table 1 presents characteristics of the RSI versus no-RSI groups of the full cohort.

\section{Propensity score-matched cohort}

A total of 38352 complete observations (including 782 RSI) were used to predict propensity scores with a boosted logistic regression model. The pseudo- $\mathrm{R}^{2}$ for the boosted propensity score model was 0.56 for the training dataset and 0.38 for the test dataset, which indicates no overfitting. After a 1:1 match, the analysis cohort contained 1454 strokes with 727 RSI matched to 727 patients with non-RSI (figure 1). Baseline characteristics of the matched cohort are provided in table 2 .

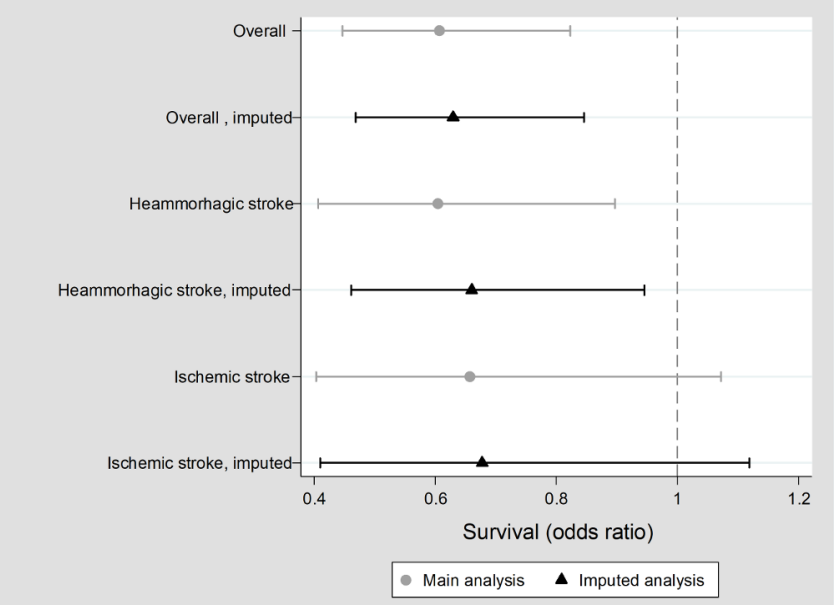

Figure 2 Survival of RSI versus no-RSI in strokes in a propensity matched cohort (dashed line at zero survival difference and with $95 \%$ Cls). RSI, rapid sequence intubation.

The patients were well matched on all relevant characteristics, and the distribution of propensity scores was similar between matched groups (online supplementary figure S2). A multilevel logistic regression analysis of the matched cohort shows that survival to hospital discharge was lower in the RSI group: OR 0.61 (95\% CI: 0.45 to $0.82 ; \mathrm{p}=0.001$ ) (figure 2). This model had good fit: Hosmer-Lemeshow $\chi^{2}(\mathrm{DF}=8 ; \mathrm{p}=0.16)$. This regression adjusted for all variables that were in the propensity score prediction model. We also added initial $\mathrm{SPO}_{2}$ and initial blood glucose levels to this model, since there were few missing data for these two variables in the matched sample, even though there were too many missing to include these in the propensity score prediction model.

The mean time to intubation from arrival at the patients side was $44.1 \mathrm{~min}(\mathrm{SD}=19.2)$ and time to intubation did not predict survival; OR 1.00 (95\% CI: 0.99 to $1.02 ; \mathrm{p}=0.54$ ). The ratio of covariate variance between the RSI and no-RSI group in the matched sample is 1.13. A final end-tidal $\mathrm{CO}_{2}$ reading of less than $35 \mathrm{~mm} \mathrm{Hg}$ was present in $43.5 \%$ of patients that had received RSI; less than $25 \mathrm{~mm} \mathrm{Hg}$ in $1.7 \%$ and higher than $45 \mathrm{~mm} \mathrm{Hg}$ in $3.6 \%$. Additionally, $12.1 \%$ of patients that received RSI had a minimum end-tidal $\mathrm{CO}_{2}$ of less than $25 \mathrm{~mm} \mathrm{Hg}$. For patients that had received RSI, $1.7 \%$ had a final systolic blood pressure of less than $90 \mathrm{~mm} \mathrm{Hg}$, and $13.3 \%$ had a minimum systolic blood pressure of less than $90 \mathrm{~mm} \mathrm{Hg}$. A final $\mathrm{SPO}_{2}$ of less than $90 \%$ was noted in $2.5 \%$, and a minimum $\mathrm{SpO}_{2}$ of less than $90 \%$ seen in $26.3 \%$ of patients that had received RSI.

We calculated the difference between the final and first measured systolic blood pressure, pulse rate, respiratory, GCS and oxygen saturation for all patients (table 3). In the matched cohort, the RSI group showed a larger decrease in systolic blood pressure than the non-RSI group, with a decrease that was more than twice as large as non-RSI. Oxygen saturation improved more in the RSI than no-RSI group. Respiratory rate was decreased almost six breaths a minute in those that received RSI, compared with those that did not. Furthermore, in the RSI group, the pulse rate change was almost 10 per minute increased, compared with no-RSI where there was a slight decrease in pulse rate. In the matched cohort those that had received RSI, 68 (9.4\%) had pneumonia compared with 38 (5.2\%) of the non-RSI: difference $4.1 \%$ (95\% CI: $1.4 \%$ to $6.7 \% ; \mathrm{p}=0.002)$. 
Table 3 Comparison of changes in vital signs in a propensity score-matched cohort of 1454 strokes

\begin{tabular}{|c|c|c|c|}
\hline Change in vital sign* & RSI (mean; SD) & No-RSI (mean; SD) & Difference $(95 \% \mathrm{Cl} ; \mathrm{p})$ \\
\hline Systolic blood pressure $(\mathrm{mm} \mathrm{Hg})$ & $-16.1(40.6)$ & $-6.4(30.6)$ & $9.7(6.0$ to $13.6 ; p<0.001)$ \\
\hline $\mathrm{SPO}_{2}(\%)$ & $4.9(11.1)$ & $3.6(10.2)$ & $-1.3(-2.5$ to $-0.06 ; p=0.04)$ \\
\hline Respiratory rate (per minute) & $-5.9(9.4)$ & $-0.2(5.8)$ & 5.6 (4.8 to $6.5 ; p<0.001)$ \\
\hline Glasgow Coma Scale (unit) & $-2.8(3.3)$ & $0.9(3.0)$ & 3.7 (3.4 to $4.1 ; p<0.001)$ \\
\hline Pulse (per minute) & $10.4(27.8)$ & $-0.9(17.4)$ & $-11.3(-13.7$ to $-8.9 ; p<0.001)$ \\
\hline
\end{tabular}

${ }^{*}$ All changes in vital signs were calculated as the last minus the first measurement on scene.

$\mathrm{RSI}$, rapid sequence intubation.

\section{Subgroup analysis comparing ischaemic versus haemorrhagic strokes}

We compared the survival of patients that had received RSI to no-RSI separately for ischaemic and haemorrhagic strokes in the matched cohort. The survival for haemorrhagic stroke was OR 0.60 (95\% CI: 0.41 to $0.90 ; p=0.01$ ) and OR 0.66 (95\% CI: 40 to $1.07 ; \mathrm{p}=0.09$ ) for ischaemic strokes (figure 2 ).

\section{Sensitivity analysis of unmeasured confounding and missing data}

Two sensitivity analyses were completed. One accounted for the impact of missing covariate data; the other was a sensitivity analysis that quantified the effect of additional unmeasured confounding. A sixth (12.5\%) of the cohort had missing data on either a covariate or outcome (figure 1). Females had more missing outcomes and those with missing outcomes were younger. Missing outcomes had a lower initial respiratory rate and a lower initial systolic blood pressure. The year 2017 had the most missing survival data compared with 2009 with the least missing. For other covariates, no significant differences were found. We imputed missing covariate data and repeated the propensity matching and analysis, which found no obvious differences in survival estimates between the imputed and main analysis (figure 2).

We estimated the impact of unmeasured confounding for an additional $10 \%$ to $100 \%$ prevalence of an illness severity confounder in the RSI group and an extra 5\% in the no-RSI group. Calculations were based on a relative risk of survival of 0.44 of this unmeasured confounder. Using a deterministic sensitivity analysis, our analysis showed that only when an extra $64 \%$ unaccounted illness severity in the RSI group is present then does survival difference become zero between RSI and the no-RSI groups (online supplementary figure S3). When an additional unmeasured illness severity beyond $64 \%$ is reached in the RSI group, estimates start favouring RSI. The measure for illness severity used in this analysis is initial GCS, and it predicted survival well with an area under curve (AUC) of 0.78 . When initial GCS is combined with the Walraven-Elixhauser comorbidity score the AUC increases to 0.82 .

\section{Limitations}

If the anticipated effect of an intervention is small, then the use of observational methods to quantify the treatment effects has limitations. In such a case, any unmeasured confounding can obscure the true effect of the treatment under evaluation. We believe this is a significant limitation with our study, as the anticipated benefit of RSI is likely to be small. At best, our results can give indication of the direction of effect of RSI in stroke, not the exact survival estimate one might get from a randomised trial. Additionally, our data did not permit a comparison of good neurological survival, which would have been a more suitable outcome.
We selected the study cohort by identifying strokes from in-hospital records, but these records did not reliably indicate if each patient was transported by ambulance. To ascertain if a patient with stroke was transported by ambulance, we had to link the in-hospital records to out-of-hospital records. Therefore, it is possible that some strokes that were transported by ambulance could not be identified by such linkage. Consequently, we could not be certain that we included the exact proportion of strokes that were taken to hospital by ambulance. However, the proportion of stroke RSI very closely match numbers from previous reports from Victoria, Australia, suggesting that this study captured most (if not all) stokes with RSI. ${ }^{3}$ Our analysis included only haemorrhagic or ischaemic strokes and not any unclassifiable strokes. Future research should investigate the effect of RSI on this subset of unclassifiable strokes. Also, the stroke diagnoses were based on ICD10-AM codes and were not adjudicated otherwise, which is less than ideal. We found differences in some covariates between those with missing survival outcomes. Although these differences reached statistical significance due to the large sample of this study, the magnitude of these differences was very small and probably clinically insignificant. Accordingly, the imputed analysis was not meaningfully different from the main analysis. We also estimate that the missing data is missing at random, based on our understanding of the data generation process, but cannot be sure of this.

It is also possible that the differences in results are due to unmeasured confounding. Unmeasured confounding due to illness severity was the main threat to the validity of our results, which could cause confounding by indication where sicker patients receive RSI, thus biassing estimates. Despite not having for-purpose illness severity scores such as NIHSS, we believe that no large unmeasured confounding is present in this study, as our measures of prognostic risk predicted survival well with an AUC of 0.82 . If a prognostic risk measure has an AUC of more than 0.75 , such an analysis is protected against confounding by illness and comorbidity risk. ${ }^{16}$

\section{DISCUSSION}

This study showed lesser odds of survival for patients that received paramedic RSI for strokes in the out-of-hospital setting. We expected that sicker patients receive more RSI, causing confounding-by-indication. If there are more patients with a poor prognosis in the RSI group, then one might find lower survival than expected for RSI. Almost three-quarters of the RSI in this study were on haemorrhagic strokes, compared with the no-RSI group which was mostly ischaemic strokes. Since haemorrhagic strokes had a poorer prognosis than ischaemic strokes, the large number of haemorrhagic strokes in the RSI group could bias results in favour of no-RSI. To counter this imbalance of prognosis between the RSI and no-RSI group, this analysis used propensity score matching. Ideally, a randomised controlled trial would be used to compare RSI to no-RSI, as this is the 
most reliable method of ensuring that confounding factors are balanced between the treated and control groups. When a trial is not easily conducted, observational research using propensity score methods can be used. Propensity score matching attempt to emulate features of a randomised trial and can lessen measured confounding. ${ }^{17}$

While our analysis is likely the first out-of-hospital study to compare RSI to no-RSI for strokes, these results are not the first to point to possible harm from intubation in stroke. Observational in-hospital studies of haemorrhagic strokes and stroke thrombectomy showed decreased survival for those that received endotracheal intubation. ${ }^{18}{ }^{19}$ Numerous causes for the association of decreased survival and intubation have been proposed. Alterations of blood pressure after laryngoscopy and pre-medications, pneumonia, hypo/hyperoxia, hypo/hyperventilation, cardiac arrhythmias, adult respiratory distress syndrome and atelectasis as well as dysphagia associated with intubation are possible causes of decreased survival. ${ }^{60-23}$ Some of these mechanisms were evident in our findings.

When scrutinising the minimum and final on-scene values of end-tidal $\mathrm{CO}_{2}$, oxygen saturation and blood pressure, it is evident that a sizeable proportion of RSI had out-of range values. However, it must be said that these minimum (as compared with the final) values were not necessarily attributable to RSI; they were measured at any time on scene. We also found that pneumonia was significantly higher in the RSI group, but it is not possible to conclude that pneumonia was caused by RSI. When looking at the final measurements of on-scene oxygen saturation, carbon dioxide and blood pressure, it is clear that adverse events were below or similar to those of out-of hospital physicians. ${ }^{24}$ Even so, these derangements associated with RSI as well as the increase in pneumonia could be factors that explain the decreased survival for RSI in this study. Future research should study the effects of vital-sign derangements in stroke.

Larynscopy might have transiently elevated blood pressure in some RSI. In a study by Perkins et al, laryngoscopy and intubation provoked a hypertensive response in $79 \%$ of their cohort, ${ }^{22}$ and it could be that RSI provoked similar response in haemorrhagic strokes, for which the blood pressure was already elevated. While RSI can transiently elevate blood pressure, our analysis shows that the final on-scene systolic blood pressure tended to be lower than the initial blood pressure. The difference between the first and final blood pressure is $9.7 \mathrm{~mm} \mathrm{Hg}$ lower for those that received RSI, compared with no-RSI. Stated differently, our analysis shows that RSI is associated with an almost $10 \mathrm{~mm} \mathrm{Hg}$ larger decrease in systolic blood pressure when compared with no-RSI. Lowering blood pressure is a likely cause of decreased survival in stroke, ${ }^{25}$ which might explain the decreased survival for strokes that received RSI in our analysis. Additionally, in the RSI group, a significant increase in pulse rate is apparent, compared with non-RSI, where the pulse rate decreased slightly. Although laryngoscopy in RSI could cause transient increase in blood pressure, we found a decrease when comparing the first to the final blood pressure. This apparent paradox indicates a complicated relationship between RSI and blood pressure that needs more clarification.

Generally, the intubation first-pass success and adverse event rate of paramedics in this study are on par with that of out-of-hospital physicians and better than other paramedic RSI studies. ${ }^{24}$ Despite the proficiency in RSI by paramedics in Victoria, Australia, our observational analysis shows decreased survival for RSI in strokes. A randomised controlled trial is urgently needed.

\section{CONCLUSIONS}

This study shows decreased survival for patients that received paramedic initiated RSI for strokes, with haemorrhagic strokes having worse survival. Derangements in carbon dioxide levels, blood pressure, oxygen saturation and increased prevalence of pneumonia following RSI might explain the decreased survival for RSI. A clinical trial comparing RSI to no-RSI in stoke is needed.

Acknowledgements We are grateful to the staff at the Centre for Victorian Data Linkage as well as Salman Sabir at Ambulance Victoria for their assistance in obtaining the data for this study.

Contributors PFF conceived of the study, KS and PF collected data and PF analysed all data. All authors contributed to the manuscript. PF takes responsibility for the paper as a whole.

Funding The authors have not declared a specific grant for this research from any funding agency in the public, commercial or not-for-profit sectors.

Competing interests None declared.

Patient consent for publication Not required.

Ethics approval This study has received ethics approval. Monash University Human Research Ethics Committee gave ethics approval for this study (ref. no. 8618).

Provenance and peer review Not commissioned; externally peer reviewed.

\section{REFERENCES}

1 GBD 2016 Causes of Death Collaborators. Global, regional, and national age-sex specific mortality for 264 causes of death, 1980-2016: a systematic analysis for the Global Burden of Disease Study 2016. Lancet 2017;390:1151-210.

2 Meyer PG, Orliaguet GA, Zerah M, et al. Emergency management of deeply comatose children with acute rupture of cerebral arteriovenous malformations. Can J Anaesth 2000:47:758-66.

3 Fouche PF, Jennings PA, Smith K, et al. Survival in Out-of-hospital Rapid Sequence Intubation of Non-Traumatic Brain Pathologies. Prehosp Emerg Care 2017;21:700-8.

4 Bernard SA, Nguyen V, Cameron P, et al. Prehospital rapid sequence intubation improves functional outcome for patients with severe traumatic brain injury: a randomized controlled trial. Ann Surg 2010;252:959-65.

5 Serri K, El Rayes M, Giraldeau G, et al. Traumatic brain injury is not associated with significant myocardial dysfunction: an observational pilot study. Scand J Trauma Resusc Emerg Med 2016;24:31.

6 Frontera JA, Fernandez A, Schmidt JM, et al. Impact of nosocomial infectious complications after subarachnoid hemorrhage. Neurosurgery 2008;62:80-7.

7 Victoria A. Clinical Practice Guidelines for Ambulance and MICA paramedics. Version 1.1 ed. Doncaster, Victoria. 2016:42-7.

8 Fouche PF, Carlson JN, Ghosh A, et al. Frequency of adjustment with comorbidity and illness severity scores and indices in cardiac arrest research. Resuscitation 2017;110:56-73

9 Sjoding MW, Luo K, Miller MA, et al. When do confounding by indication and inadequate risk adjustment bias critical care studies? A simulation study. Critical Care 2015:19:195.

10 van Walraven C, Austin PC, Jennings A, et al. A modification of the Elixhauser comorbidity measures into a point system for hospital death using administrative data. Med Care 2009:47:626-33.

11 Mansour OY, Megahed MM, Abd Elghany EHS. Acute ischemic stroke prognostication, comparison between Glasgow Coma Score, NIHS Scale and Full Outline of UnResponsiveness Score in intensive care unit. Alexandria Journal of Medicine 2015:51:247-53.

12 Montgomery JM, Nyhan B, Torres M. How conditioning on posttreatment variables can ruin your experiment and what to do about It. Am J Pol Sci 2018;62:760-75.

13 Hastie T, Tibshirani R, Friedman JH. The elements of statistical learning: data mining, inference, and prediction. New York: Springer, 2001.

14 Greenland S. Basic methods for sensitivity analysis of biases. Int J Epidemiol 1996:25:1107-16.

15 Kyriacou DN, Lewis RJ. Confounding by indication in clinical research. JAMA 2016;316:1818-9.

16 Sjoding MW, Luo K, Miller MA, et al. When do confounding by indication and inadequate risk adjustment bias critical care studies? A simulation study. Crit Care 2015; 19:195.

17 Kitsios GD, Dahabreh IJ, Callahan S, et al. Can we trust observational studies using propensity scores in the critical care literature? A systematic comparison with randomized clinical trials. Crit Care Med 2015;43:1870-9.

18 Takahashi CE, Brambrink AM, Aziz MF, et al. Association of intraprocedural blood pressure and end tidal carbon dioxide with outcome after acute stroke intervention. Neurocrit Care 2014;20:202-8 
19 Khatri P, Yeatts SD, Mazighi M, et al. Time to angiographic reperfusion and clinical outcome after acute ischaemic stroke: an analysis of data from the Interventional Management of Stroke (IMS III) phase 3 trial. Lancet Neurol 2014;13:567-74.

20 Froehler MT, Fifi JT, Majid A, et al. Anesthesia for endovascular treatment of acute ischemic stroke. Neurology 2012;79(13 Suppl 1):S167-S173.

21 Jeon SB, Choi HA, Badjatia N, et al. Hyperoxia may be related to delayed cerebral ischemia and poor outcome after subarachnoid haemorrhage. J Neurol Neurosurg Psychiatry 2014;85:1301-7.
22 Perkins ZB, Gunning M, Crilly J, et al. The haemodynamic response to pre-hospital RSI in injured patients. Injury 2013;44:618-23.

23 Takahashi C, Liang CW, Liebeskind DS, et al. To tube or not to tube? The role of intubation during stroke thrombectomy. Front Neurol 2014;5.

24 Fouche PF, Stein C, Simpson P, et al. Nonphysician out-of-hospital rapid sequence intubation success and adverse events: a systematic review and meta-analysis. Ann Emerg Med 2017;70:449-59.

25 Johnston KC, Durkalski-Mauldin VL. Considering prehospital stroke trials: Did right-2 get it right? Lancet 2019;393:963-5. 